\title{
CLOSED GRAPHS AND CLOSED PROJECTIONS ${ }^{1}$
}

\section{T. SCARBOROUGH}

If $X$ and $Y$ are topological spaces, we say that the pair $(X, Y)$ has the closed graph property (C.G.P.) if every function on $A \subseteq X$ into $Y$ with a closed graph $G(f)$ in $X \times Y$ is continuous on $A$. We say that the pair $(X, Y)$ has the closed projection property (C.P.P.) if the projection $\pi_{1}$ of $X \times Y$ onto $X$ is a closed function, i.e. if $\pi_{1} C$ is closed for all closed subsets $C$ of $X \times Y$. If $\pi_{1}$ maps the closures of open sets onto closed sets, then we say that the pair $(X, Y)$ has the regular closed projection property (R.C.P.P.). A space $X$ is said to be $H(i)$ if every open filter base on $X$ has nonvoid adherence.

If $Y$ is compact, then $(X, Y)$ has C.G.P. and C.P.P. for all spaces $X$. Both of these results are well known, e.g. see [2, pp. 228-229]. Also, a discussion of C.G.P. can be found in [10]. If $Y$ is $H(i)$, then $(X, Y)$ has R.C.P.P. by [8, p. 136]. Closed graphs, closed projections, closed relations and the relation of these properties to various compactness conditions have been studied in [3], [5], [6], [7] and [8].

It is the purpose of this paper to investigate C.G.P., C.P.P. and R.C.P.P., and to elaborate on and extend some of the results in the papers mentioned above. We also show that the properties feeble compactness or light compactness, and R.C.P.P. are closely related.

The closure of a set $A$ will be denoted by $A^{\prime}$, and $\pi_{1}$ will denote the first projection mapping. Inclusion will be denoted by $\subseteq$ and proper inclusion by $C$. The positive integers will be denoted by $I$.

The author wishes to thank the referee for bringing several references to his attention, particularly [3], which seems to overlap somewhat with this paper, and for improving Theorem 5 and Corollary 6 .

1. THEOREM. For each space $Y$, there exists a zero dimensional hereditarily paracompact Hausdorff space $Y^{*}$ such that: (1) If $Y$ is $T_{1}$ and $\left(Y^{*}, Y\right)$ has C.G.P., then $Y$ is compact. (2) If $\left(Y^{*}, Y\right)$ has C.P.P., then $Y$ is compact.

Let $\mathfrak{F}=\left\{\mathfrak{F}_{a}: a \in A\right\}$ be the set of all ultrafilters on $Y$. Each $\mathfrak{F}_{a} \in \mathfrak{F}$ is a directed set with respect to reverse inclusion. Thus $\prod\left\{\mathcal{F}_{a}: a \in A\right\}$ is a directed set with the ordering $\left\langle F_{a}\right\rangle \leqq\left\langle G_{a}\right\rangle$ if $\left\langle F_{a}\right\rangle,\left\langle G_{a}\right\rangle \in \prod\left\{\xi_{a}: a \in A\right\}$ and $G_{a} \subseteq F_{a}$ for all $a \in A$. We define a topology on the power set $Y^{*}$ of $Y$ by letting each point of $Y^{*}-\{\varnothing\}$ be open, and by taking 1968.

Presented to the Society, January 26, 1968; received by the editors February 12,

1 This research was partially supported by a grant from the State of Mississippi. 


$$
N\left\langle F_{a}\right\rangle=\cup\left\{\left\{F \in F_{a}: F \subset F_{a}\right\}: a \in A\right\} \cup\{\varnothing\}
$$

to be an open neighborhood of $\varnothing$ for each $\left\langle F_{a}\right\rangle$ in $\prod\left\{F_{a}: a \in A\right\}$.

In order to verify that $Y^{*}$ is Hausdorff, it is sufficient to show that if $z^{*} \neq \varnothing$, then there exists an $N\left\langle F_{a}\right\rangle$ such that $z^{*} \notin N\left\langle F_{a}\right\rangle$. If $z^{*} \in F_{a}$, let $F_{a}=z^{*}$. If $z^{*} \notin F_{a}$, then, since each $\Im_{a}$ is an ultrafilter, there exists an $H \in \mathcal{F}_{a}$ such that $H \cap z^{*}=\varnothing$; in this case let $F_{a}=H$. It is clear from the definition of $N\left\langle F_{a}\right\rangle$ that $z^{*} \notin N\left\langle F_{a}\right\rangle$, so $Y^{*}$ is Hausdorff. Since every point of $Y^{*}-\{\varnothing\}$ is open in $Y^{*}$, every point of $Y^{*}$ has arbitrarily small neighborhoods with empty boundary, so $Y^{*}$ is zero dimensional. The verification that $Y^{*}$ is hereditarily paracompact is routine and is left to the reader.

If $Y$ is not compact, then there exists an ultrafilter $\mathcal{F}$ on $Y$ with void adherence. For each $F \in \mathcal{F}$, choose $x(F) \in F$ and consider the set $\left.C=\cup\{F\} \times\{x(F)\}^{\prime}: F \in \mathcal{F}\right\}$. We will show that $C$ is closed in $Y^{*} \times Y$. Suppose $\left(y^{*}, y\right) \notin C$. If $y^{*}=F \in \mathcal{F}$, then $y \notin\{x(F)\}^{\prime}$, so there exists a neighborhood $U$ of $y$ such that $x(F) \notin U$. Thus $\left\{y^{*}\right\} \times U$ is a neighborhood of $\left(y^{*}, y\right)$ which fails to meet $C$. If $y^{*} \notin \mathscr{F}$ and $y^{*} \neq \varnothing$, then $\left\{y^{*}\right\} \times Y$ is a neighborhood of $\left(y^{*}, y\right)$ which is disjoint from $C$. Suppose $y^{*}=\varnothing$. The filter $\mathcal{F}$ has void adherence, so there exists a $G \in \mathcal{F}$ and an open neighborhood $U$ of $y$ such that $G \cap U=\varnothing$. Choose a neighborhood $N\left\langle F_{a}\right\rangle$ of $\varnothing$ such that $z^{*}=G \notin N\left\langle F_{a}\right\rangle$ in exactly the same way as was done in the verification that $Y^{*}$ was Hausdorff. We will show that $N\left\langle F_{a}\right\rangle \times U$ is disjoint from $C$. Let $F \in \mathcal{F}$. If $F \subseteq G$, then $x(F) \in G$, so $x(F) \notin U$. Thus

$$
\left(N\left\langle F_{a}\right\rangle \times U\right) \cap\left(\{F\} \times\{x(F)\}^{\prime}\right)=\varnothing .
$$

Suppose $F \nsubseteq G$ and $F \in \mathcal{F}_{a}$. If $G \in \mathcal{F}_{a}$, then $F_{a}=G$ and $F \notin\left\{F \in \mathcal{F}_{a}: F \subset F_{a}\right\}$. If $G \notin \mathcal{F}_{a}$, then $F_{a} \cap G=\varnothing$. Since $F$ and $G$ are both in $\Im, F \cap G \neq \varnothing$. Thus $F \Phi F_{a}$, so $F \notin\left\{F \in F_{a}: F \subset F_{a}\right\}$. Therefore

$$
\left(N\left\langle F_{a}\right\rangle \times U\right) \cap\left(\{F\} \times\{x(F)\}^{\prime}\right)=\varnothing .
$$

Since $F$ was arbitrary in $\mathcal{F}$, it follows that $\left(N\left\langle F_{a}\right\rangle \times U\right) \cap C=\varnothing$, so $C$ is closed.

(1) Suppose $\left(Y^{*}, Y\right)$ has C.G.P. Let $f=C \cup\{(\varnothing, y)\}$ where $y$ is any point of $Y$. Since $Y^{*}$ and $Y$ are $T_{1}$ spaces, $f$ is a function with a closed graph in $Y^{*} \times Y$. Thus, by the hypothesis, $f$ is continuous on $\mathscr{F} \cup\{\varnothing\}$. However, $f$ cannot be continuous at $\varnothing ; \mathcal{F}$ (considered as a net in $Y^{*}$ ) converges to $\varnothing$ and $\{x(F): F \in \mathcal{F}\}$ has no cluster points since $\mathscr{F}$ has void adherence. This is a contradiction.

(2) Suppose $\left(Y^{*}, Y\right)$ has C.P.P. If $Y$ is not compact, we simply observe that $C$ is closed, $\mathcal{F}$ converges to $\varnothing, \mathcal{F} \subseteq \pi_{1} C$ and $\varnothing \notin \pi_{1} C$. 
The next two corollaries are now obvious, so their proofs are omitted.

2. Corollary. Let $Y$ be a $T_{1}$ space. Then $Y$ is compact if and only if $\left(Y^{*}, Y\right)$ has C.G.P. Also, $Y$ is compact if and only if $(X, Y)$ has C.G.P. for all spaces $X$.

3. Corollary. A space $Y$ is compact if and only if $\left(Y^{*}, Y\right)$ has C.P.P. Also, $Y$ is compact if and only if $(X, Y)$ has C.P.P. for all spaces $X$.

The second assertion of Corollary 2 (Corollary 3 ) also follows from $[5$, p. 436] ([7, p. 21]). The second assertion of Corollary 2 was known to the author prior to the publication of [5].

In (1), the hypothesis that $Y$ be $T_{1}$ is more or less essential, since if $Y$ is the range of any function with a closed graph, then $Y$ is $T_{1}$; see [10, p. 198].

It is well known [2, p. 228] that C.P.P. implies C.G.P. Thus (1) of Theorem 1 implies (2) of Theorem 1 whenever $Y$ is $T_{1}$. It is not true that C.G.P. implies C.P.P.; see Example 6. However, with suitable restrictions, we are able to obtain a partial converse.

From [4], a set $U \subset X$ is said to be sequentially open iff every sequence in $X$ converging to a point in $U$ is eventually in $U$. The space $X$ is said to be sequential iff every sequentially open subset of $X$ is open.

4. Theorem. Let $X$ be sequential $T_{1}$ and $Y$ be $T_{1}$. If $(X, Y)$ has C.G.P., then $(X, Y)$ has C.P.P.

Suppose $\pi_{1} C$ is not closed where $C$ is a closed subset of $X \times Y$. Then $X-\pi_{1} C$ is not sequentially open, so there exists an $x \in X-\pi_{1} C$ and a function $S=\left\{\left(x_{n}, y_{n}\right): n \in I\right\}$ in $C$ such that $\left\{x_{n}: n \in I\right\}$ converges to $x$. Let $y$ be any point of $Y$ and $G(f)=\{(x, y)\} \cup S$. It is clear that $f$ is a function on $\{x\} \cup\left\{x_{n}: n \in I\right\}$ into $Y$. To show $G(f)$ is closed, it suffices to prove $S$ is closed. Let $(s, t) \notin S$. Since $t$ is not a cluster point and since $Y$ is $T_{1}$, there exists a neighborhood $V$ of $t$ such that $V \cap\left\{y_{n}: n \in I\right\}=\varnothing$ or $V \cap\left\{y_{n}: n \in I\right\}=\left\{y_{k}\right\}$. In the first case $(X \times V) \cap S=\varnothing$. In the second case $s \notin\left\{x \in X:\left(x, y_{k}\right) \in S\right\}$, so we can choose a neighborhood $U$ of $s$ which is disjoint from $\{x \in X$ : $\left.\left(x, y_{k}\right) \in S\right\}$; thus, $(U \times V) \cap S=\varnothing$. Therefore, $S$ is closed and from the hypothesis, $f$ must be continuous at $x$. But this is impossible since $\left\{y_{n}: n \in I\right\}$ has no cluster points. Thus $\pi_{1} C$ is closed and $\pi_{1}$ is a closed function.

5. Corollary. Let $X$ be a sequential nondiscrete $T_{1}$ space and let $Y$ 
be $T_{1}$. Then the following conditions are equivalent: (1) $(X, Y)$ has C.G.P. (2) $(X, Y)$ has C.P.P. (3) $Y$ is countably compact.

That (1) and (2) are equivalent follows from Theorem 4 and the fact that C.P.P. implies C.G.P.; (2) implies (3) is a consequence of [6, Theorem 4] and (3) implies (2) follows from [3].

The author wishes to thank the referee for pointing out that the hypothesis in Theorem 5 and Corollary 6 could be weakened from first countable to sequential.

The following is an example of a first countable $T_{0}$ space $X$ with the following properties: (1) If $Y$ is any space and $f$ is a function from any subset of $X$ into $Y$ with a closed graph, then $f$ is constant. (2) If $Y$ is not countably compact, then $(X, Y)$ does not have C.P.P. Thus the hypothesis on $X$ in Theorem 4 cannot be weakened from $T_{1}$ to $T_{0}$.

6. Example. Let $X=\left\{x_{0}, x_{1} \cdots\right\}$ be a countable set. Let $A_{n}=\left\{x_{j}: j \geqq n\right\} \cup\left\{x_{0}\right\}$ and define a base for a topology on $X$ as follows: $\Re\left(x_{0}\right)=\left\{A_{n}: n=1,2, \cdots\right\}$ and $\mathscr{N}\left(x_{n}\right)=\left\{A_{n}\right\}$ for $n \geqq 1$. The details are left to the reader.

The referee has raised the question as to whether C.G.P. implies C.P.P. when both spaces are Hausdorff. As far as I know, this is an open problem.

7. THEOREM. For each space $Y$, there exists a zero dimensional hereditarily paracompact Hausdorff space $T^{*}$ such that if $\left(T^{*}, Y\right)$ has R.C.P.P., then $Y$ is $H(i)$.

Let $\mathfrak{I}$ be the topology on $Y$ and let $\mathfrak{T}=\left\{\Im_{a}: a \in A\right\}$ be the set of all maximal open filter bases on $Y$. As in Theorem $1, \prod\left\{F_{a}: a \in A\right\}$ is a directed set. We define a topology on the set $T^{*}=\mathfrak{J}$ by letting each point of $T^{*}-\{\varnothing\}$ be open and by taking

$$
N\left\langle F_{a}\right\rangle=\cup\left\{\left\{F \in F_{a}: F \subset F_{a}\right\}: a \in A\right\} \cup\{\varnothing\}
$$

to be an open neighborhood of $\varnothing$ for each $\left\langle F_{a}\right\rangle$ in $\prod\left\{F_{a}: a \in A\right\}$.

That $T^{*}$ is Hausdorff, zero dimensional, and hereditarily paracompact is a verbatim repetition of the corresponding argument given for $Y^{*}$ except that ultrafilters are replaced by maximal open filter bases.

Suppose $\left(T^{*}, Y\right)$ has R.C.P.P. If $Y$ is not $H(i)$, then there exists a maximal open filter base $\mathcal{F}$ with void adherence. Let $C$ $=\bigcup\{\{F\} \times F: F \in \mathcal{F}\}$. Then $C$ is open since each $\{F\} \times F$ is open. By an argument similar to that given in Theorem 1, we can show that 
$\cup\left\{\{F\} \times F^{\prime}: F \in \mathcal{F}\right\}$ is closed; thus $C^{\prime}=U\left\{\{F\} \times F^{\prime}: F \in \mathcal{F}\right\}$. But $\varnothing \in\left(\pi_{1} C\right)^{\prime}-\pi_{1} C$. This is a contradiction.

8. Corollary. Let $Y$ be any space. Then $Y$ is $H(i)$ if and only if $\left(T^{*}, Y\right)$ has R.C.P.P. Also $Y$ is $H(i)$ if and only if $(X, Y)$ has R.C.P.P. for all spaces $X$.

The proof, which follows from the preceding theorem and $[8$, p. 136], is omitted.

A space $X$ is said to be feebly compact or lightly compact if every locally finite system of open sets of $X$ is finite. These spaces have been studied in [1], [8, \$4] and [9, p. 901]. In [1, Theorem 1], it is shown that a space $X$ is feebly compact if and only if every countable open covering of $X$ possesses a finite subset $A_{1}, \cdots, A_{n}$ whose closures cover $X$, i.e. $X \subset\left\{A_{i}^{\prime}: 1 \leqq i \leqq n\right\}$. It follows easily that a space $X$ is feebly compact if and only if every countable open filter base on $X$ has nonvoid adherence. This extends the list of equivalences given in [1, Theorem 1].

Using this characterization of feebly compact spaces, we are able to obtain

9. Theorem. If $X$ is first countable and $Y$ is feebly compact, then $(X, Y)$ has R.C.P.P.

Let $A$ be open in $X \times Y$ and $x \in\left(\pi_{1} A\right)^{\prime}$. Since $X$ is first countable, there exists an open countable neighborhood base $\left\{U_{n}\right\}$ of $x$. Since $Y$ is feebly compact, the countable open filter base $\mathcal{F}=\left\{\pi_{2}\left(U_{n} \times Y \cap A\right)\right.$ : $n=1,2, \cdots\}$ has a point of adherence, say $y$. It follows that $(x, y) \in A^{\prime}$, so $x \in \pi_{1}\left(A^{\prime}\right)$. Thus $\pi_{1}\left(A^{\prime}\right)$ is closed.

10. Corollary. If $X$ is first countable and $Y$ is completely regular and pseudo-compact, then $(X, Y)$ has R.C.P.P.

This follows from [1, Theorem 3] and the preceding theorem.

11. TheOREM. Let $Y$ be an arbitrary space and let $X$ be a space containing a point $x$ with an open neighborhood system $\left\{U_{n}: n=1,2, \cdots\right\}$ such that $U_{n} \supset U_{n+1}^{\prime}$ for all $n$. Then $Y$ is feebly compact if $(X, Y)$ has R.C.P.P.

If $Y$ is not feebly compact, then there exists an open countable filter base $\left\{F_{n}\right\}$ on $Y$ with void adherence. For each $n$, let $G_{n}=\bigcap\left\{F_{k}\right.$ : $1 \leqq k \leqq n\}$ and let

$$
A=U\left\{\left(U_{n}-U_{n+1}^{\prime}\right) \times G_{n}: n=1,2, \cdots\right\} .
$$


Clearly $A$ is an open subset of $X \times Y$. It is easy to verify that $x \in\left(\pi_{1} A\right)^{\prime}-\pi_{1}\left(A^{\prime}\right)$, so $\pi_{1}$ is not closed. This is a contradiction.

12. Corollary. Let $X$ satisfy the hypothesis of Theorem 11. If $Y$ is completely regular and $(X, Y)$ has R.C.P.P. then $Y$ is pseudocompact.

This follows from [1, Theorem 3].

13. Theorem. Let $X$ be a nonempty first countable regular nondiscrete $T_{1}$ space and let $Y$ be an arbitrary space. Then $(X, Y)$ has R.C.P.P. if and only if $Y$ is feebly compact.

"If" follows from Theorem 9 and "only if" follows from Theorem 11.

The final result is an immediate consequence of Theorem 13 and $[1$, Theorem 3].

14. Corollary. Let $X$ be a first countable regular nondiscrete $T_{1}$ space and let $Y$ be completely regular. Then $(X, Y)$ has R.C.P.P. if and only if $Y$ is pseudocompact.

\section{REFERENCES}

1. R. W. Bagley, E. H. Connell and J. D. McKnight, Jr., On properties characterizing pseudo-compact spaces, Proc. Amer. Math. Soc. 9 (1958), 500-506.

2. J. Dugundji, Topology, Allyn and Bacon, Boston, Mass., 1966.

3. Isidore Fleischer and S. P. Franklin, On compactness and projections (to appear).

4. S. P. Franklin, Spaces in which sequences suffice, Fund. Math. 5 (1965), 107-116.

5. S. P. Franklin and R. H. Sorgenfrey, Closed and image-closed relations, Pacific J. Math. 19 (1966), 433-439.

6. S. Hanai, Inverse images of closed mappings. I, Proc. Japan Acad. 37 (1961), 298-301.

7. S. Mrowka, Compactness and product spaces, Colloq. Math. 7 (1959), 19-22.

8. C. T. Scarborough and A. H. Stone, Products of nearly compact spaces, Trans. Amer. Math. Soc. 124 (1966), 131-147.

9. A. H. Stone, Hereditarily compact spaces, Amer. J. Math. 82 (1960), 900-916.

10. A. Wilansky, Functional analysis, Blaisdell, New York, 1964.

Mississippi State University 